XXVIII.-On the Preparation of Chrysammic Acid.

\author{
By Joh N TEn house, LL.D., F.R.S., \&c., and Huqo Mü LLER, \\ PhD., F.R.S., \&c.
}

The preparation of chrysarnmic acid is both troublesome and tedious. We think, however, that the following will be found more advantageous than any of the processes which have hitherto been published. Although at first sight this process may appear complicated, yet it is not more so than is necessary to obtain a good result; for if the action of the nitric acid be not pushed far enough, the large quantity of aloetic acid and resinous matter unconverted is a great hindrance to the purification of the chrysammic acid; whilst, on the other hand, if the action be continued too long, much of the chrysammic acid is destroyed. Six parts by measure of nitric acid, sp. gr. 1·36, are placed in a large retort connected with a good condensing arrangement, and when heated nearly to boiling, two parts of socotrine aloes broken into small pieces are gradually added. The action is very violent, large quantities of nitrous fumes being given off, so that at the commencement of the operation, the aloes must be added slowly, and little or no heat applied. When, however, a considerable quantity has been introduced, it will be found necessary to heat, in order to accelerate the action, and the acid which distils over should be poured back into the retort at intervals. When the whole of the aloes is in the retort, the digestion is continued for about ten hours, during the last three of which the weak acid may be distilled off, so that the contents of the retort are reduced to one-half of their original volume. To this residue three measures of nitric acid are gradually added, and the digestion is continued for about six or seven hours, distilling off the bulk of the acid during the latter part of the digestion. The residue in the retort is now poured into about four parts of water, and after agitation the undissolved picric and aloetic acids,--separated by this means from the oxalic acid produced in the reaction,--are collected, and when dry, or nearly so, introduced into a retort with one part of strong nitric acid, sp. gr. 1*45, and digested in the manner previously described for six or eight hours. The residue, which now consists of picric, aloetic, and chrysammic acids, is washed by decantation with successive quantities of boiling water, until the washings become of a 
pink colour, instead of the deep orange which they had at first. By this means the picric acid is removed. The mixture of chrysammic and aloetic acids thus obtained is dried and returned to the retort with one part more of strong nitric acid, and digested as above for ten hours, by which means most of the aloetic acid is converted into chrysammic acid.

It is now washed with hot water until the washings have a pink colour, and then boiled for a few minutes with about four parts of water and filtered. This operation is repeated three or four times, until the colour of the filtrate is bright red instead of purple.

It is then again boiled with water, and a slight excess of chalk added, when the contents of the flask become of a deep-red or purple colour. On cooling, minute red needles of chrysammate of calcium may be seen on the side of the flask, also a flocculent mass of them at the bottom. These should be collected, dried, and crystallised from dilute spirit-equal parts of alcohol and water. If the aloes have not been sufficiently acted upon, and a considerable quantity of aloetic acid is present, these needles do not crystallize out the first time, but by boiling with fresh quantities of water, and leaving the liquid to cool between each operation, they at last make their appearance, the aloetate of calcium which seems to prevent their crystallisation being removed, as it is much more soluble in cold water than the chrysammate. The red washings, \&c., obtained in these various operations, when strongly acidulated with nitric acid, yield a considerable quantity of crude aloetic acid, which may be converted into chrysammic acid by further treatment with strong nitric acid. By this process aloes yield from three to four per cent. of chrysammate of calcium. Although aloes is the most convenient source, yet considerable quantities of chrysammic acid may be economically made by treating with nitric acid the resin of aloes left undissolved in the preparation of cold extract of aloes. Though this resin yields but little more than half the amount that aloes itself does, yet being a waste product, it is an advantageous source of the acid. As already mentioned in a short paper by Warren de la Rue and Hugo Müller on some derivatives of chrysophare (Zeitschrift $f$. Chemie, 1862),* chrysammic acid is also the product of the action

* In this paper the authors point out the probable connection which exists between chrysophane and chrysammic acid; the latter being the only product of the action of fuming nitric acid on chrysophane. It might, therefore, be considered as tetranitro- 
of nitric acid on chrysophane and some other constituents of rhubarb.

Chrysammate of Calcium.

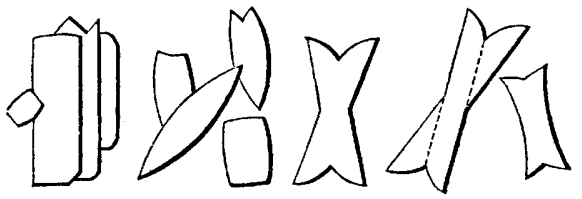

The crude chrysammate of calcium obtained by the above process is purified by crystallising several times alternately from boiling water and spirit. The clear red solution of the pure salt in boiling water forms on cooling a magma of bright red needles, which contract considerably on drying. It is very soluble in boiling alcohol, moderately so in boiling water, from which it crystallises out almost entirely on cooling. When dried in vacuo, it loses water, and becomes of a chocolate colour, but on exposure to the air, it quickly absorbs moisture and regains its original bright red colour. At $145^{\circ}$ it is anhydreus, and when analysed gave the following results :-

I. 1.021 grammes gave $\cdot 224$ grm. carbonate of calcium.

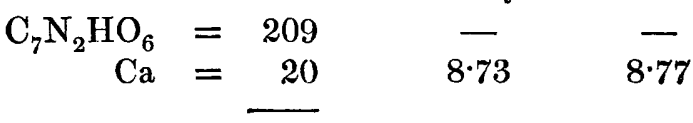

$$
\begin{aligned}
& 229
\end{aligned}
$$

Messrs. Schunck and Mulder described the chrysammate of calcium, which they obtained as a dark-red, somewhat crystalline powder.

Chrysammic Acid.

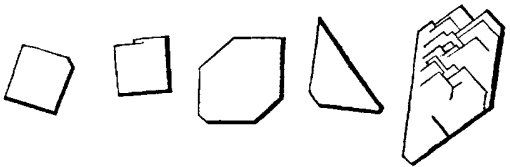

On adding a slight excess of nitric acid to a concentrated chrysophane, and as such the formula of chrysammic acid would require to be doubled. The results of the analysis of ehrysophane and chrysammic acid hitherto obtained do not however show sufficient accordance to justify already the adoption of their theory. 
boiling solution of pure chrysammate of calcium, pure chrysammic acid is precipitated. When cold the acid may be collected, and forms golden scales of considerable size and great lustre, very much resembling iodide of lead in appearance. The filtrate is quite colourless, and does not contain a trace of chrysammic acid. When suspended in water, it decomposes neutral solutions of nitrate of copper, acetate of copper, sulphate of nickel, chloride of manganese, and other salts, even at ordinary temperatures. When dried at $100^{\circ}$ and subjected to analysis, it gave the following results :-

I. 296 grm. gave $\cdot 433$ grm. carbonic acid, and 030 grm. water.

II. 2907 grm. gave 44323 grm. carbonic acid, and $\cdot 0395$ grm. water.

\begin{tabular}{lrrcc} 
& & Theory. & \multicolumn{1}{c}{ I. } & II. (H. M.) \\
$\mathrm{C}_{7}=$ & 84 & $40 \cdot 00$ & $39 \cdot 90$ & 40.57 \\
$\mathrm{~N}_{2}=$ & 28 & $13 \cdot 33$ & - & - \\
$\mathrm{H}_{2}=$ & 2 & .95 & $1 \cdot 12$ & 1.5 \\
$\mathrm{O}_{6}=$ & 96 & $45 \cdot 72$ & - & -
\end{tabular}

Chrysammic acid, when digested with a mixture of chlorate of potassium and hydrochloric acid, is slowly decomposed into chloropicrin, but without the formation of chloranil.

In the paper by Warren de la Rue and Hugo Müller, it is mentioned that when chrysammic acid is crystallised from glacial acetic acid, it takes up 21.5 to $21 \cdot 6$ per cent. of this acid, forming scaly prismatic crystals, which remain unchanged over sulphuric acid or caustic lime, but lose their acetic acid when heated above $100^{\circ} \mathrm{C}$.

\section{Benzoyl-chrysammic Acid.}

Chrysammic acid, when heated together with chloride of benzoyl, takes up benzoyl, forming a yellow compound, which crystallises in the prismatic form, and is not acted upon at ordinary temperatures by carbonates or caustic alkalies, but when treated with alcoholic potash, is decomposed, with formation of chrysammate anả benzoate of potassium. Benzoyl-chrysammic acid is nearly insoluble in most of the usual solvents. 


\section{Chrysammate of Magnesium.}
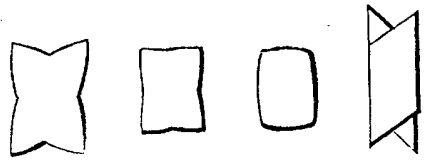

This salt is easily obtained by boiling pure chrysammic acid with an excess of magnesia, or the carbonate, for some time, filtering, and leaving the liquid to cool, when the magnesium-salt crystallises out, if the solution be not too dilute, in very fine broad red plates, having great lustre. It is by far the most beautiful of the salts of chrysammic acid.

When heated on platinum-foil it decomposes with feeble explosion, throwing off the magnesia in a fine flocculent state, in the same manner that metallic magnesium does when burning.

Dried at $160^{\circ}$ and subjected to analysis, it gave the following results :-

0.3215 grm. substance gave 0.0808 pyrophosphate of magnesium.

Theory. Analysis.

$$
\begin{aligned}
& \mathrm{C}_{7}=84 \\
& \mathrm{~N}_{2}=28 \\
& \mathrm{H}=1 \\
& \mathrm{M}^{\prime \prime \prime}=12 \\
& \mathrm{O}_{6}=\frac{96}{221}
\end{aligned}
$$$$
5 \cdot 43
$$

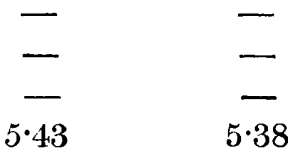

Other Salts of Chrysammic Acid.

The chrysammate of copper is even more soluble in alcohol than the calcium-compound, and may be used with advantage in the preparation and purification of chrysammic acid. It forms prismatic crystals of considerable size.

The manganese-salt crystallises from a hot aqueous solution in large, but very thin, scaly crystals. Of all the chrysammates prepared, this salt possesses the most brilliant golden lustre. By transmitted light it is blood-red.

Most of the chrysammates exhibit a considerable degree of solubility in weak alcohol, and are obtained from this solvent in 
distinct crystals, which, however, are generally very thin and scaly, so that their form cannot be ascertained with exactness. It has been observed that most of the salts of chrysammic acid are identical in form when examined under the microscope; generally they appear in the well known twin combinations so characteristic of gypsum.

\section{Hydrochrysammide.}

In addition to the processes already known for preparing this beautiful body, it may very readily be obtained by digesting pure chrysammic acid with zinc and a dilute acid, or by the action of hydriodic acid, to which a small piece of phosphorus has been added to prevent the separation of free iodine. Also on bringing chrysammic acid suspended in water in contact with sodiumamalgam, a purple solution containing hydrochrysammide is formed; and when this is shaken up in a well closed bottle with an excess of the amalgam, the purple colour soon changes into orange, but on admitting even the smallest amount of air, the purple colour is restored. 